\title{
The Research on Face Recognition and Segmentation Based on Intelligent Background
}

\author{
Jiangtao Wang \\ College of Network Communication, Zhejiang Yuexiu University of Foreign Languages, Shaoxing, China \\ Email address: \\ 3068525606@qq.com \\ To cite this article: \\ Jiangtao Wang. The Research on Face Recognition and Segmentation Based on Intelligent Background. Journal of Electrical and Electronic \\ Engineering. Vol. 8, No. 1, 2020, pp. 36-41. doi: 10.11648/j.jeee.20200801.16
}

Received: February 29, 2020; Accepted: April 1, 2020; Published: April 14, 2020

\begin{abstract}
Affected by factors such as attitude, light, expression, etc., it is impossible to accurately identify the identity in a wireless visual sensor network in an uncontrollable environment. In traditional visual identity recognition, it is necessary to convert uncontrollable factors into controllable and stable feature factors for identity recognition in a relatively uncontrollable environment where the node distribution is relatively complicated. The conversion process leads to long recognition time and low efficiency. An adaptive recognition method for identity features in wireless visual sensing networks based on LBP face recognition is proposed. A strong classifier is obtained for cascading, and the underlying features are extracted. The final Harr face cascade classifier is applied to the face Check it out. The PCA dimensionality reduction processing of the facial area feature vector is performed to obtain the low-dimensional feature vector, the dimensionality reduction coefficient, and the average face of the person. For the face image in the wireless local area, its LBP operation is given. Perform histogram statistics on face feature information, obtain face LBP histograms, and perform feature matching on the face feature database to complete recognition. The improved algorithm has improved the cumulative matching score of traditional algorithms by $17.8 \%$; the accuracy rate has improved It is $32.7 \%$, and the recognition time is shortened by $3.9 \mathrm{~s}$. Simulation results show that the proposed algorithm has high accuracy and recognition efficiency.
\end{abstract}

Keywords: Wireless Sensor, Dimensionality Reduction, Feature Matching, Adaptive Recognition

\section{Introduction}

As wireless visual sensor network development and the application of information security people put forward higher requirements [1]. Licensing is one of the important means to ensure information security, it may be advantageous to protect users of the system have the corresponding authority $[2,3]$. Identity adaptive knowledge is the key to the theoretical basis of authorization, recognition algorithm research topics have become the focus of scholars in the field, it has been more and more attention $[4,5]$.

Identity recognition technology as a key development issues of network security, common identity recognition technology including face recognition, iris recognition, fingerprint recognition. Correlation algorithm achieved certain results. Among them, the literature [6] Proposed single feature identity recognition algorithm, based on a certain facial features to identify the identity of the algorithm has fast calculation speed, real-time advantage, but the affected light, rotation, etc., in a complex context, recognition rate will be reduced; literature [7] Proposed identity recognition algorithm combined features will not be fixed in accordance with the same characteristics as the principles together, and this algorithm is more efficient overall, but the information is too large, resulting in reduced real-time recognition; literature [8] Proposed common vector combined identity 2 DPCA special algorithm, through Uram-Schmidt orthogonal transformation implemented on the same vector image is calculated, based on the results obtained to identify the minimum distance detection, the algorithm has a good discrimination function, but complexity is too high, low recognition efficiency; literature [9] Proposed a three-dimensional model of facial expression recognition algorithm identity of deformed face implementation model registration, high accuracy, there are common face model and real-time problem of poor matching model registration it can not be obtained; literature [10] SFM's proposed identity recognition algorithm, the first search match point, after SFM algorithm determined the three-dimensional 
coordinates of the feature points, using a multi-resolution image mosaic texture image forming algorithm, while the maps them to three-dimensional model, the algorithm increases the real sense, but a three-dimensional reconstruction of a large amount of computation, greatly affected the performance of its recognition.

Proposed LBP face discrimination in wireless visual sensor network identity characteristic adaptive algorithm to identify, by Harr face cascade classifier to detect human faces, human face region feature vector PCA dimension reduction, for wireless local area face images, use LBP get facial feature information obtained facial feature information histogram statistics, get face LBP histogram, which will feature visual and wireless sensor networks signatures were human face match, to complete the identification of the identity. Experimental results demonstrate that the proposed algorithm has high recognition accuracy and efficiency.

\section{Visual Identity Adaptive Wireless Sensor Networks Recognition Principle}

When identity adaptive recognition through the inspection window in a customized location adjacent rectangular matrix of each pixel is obtained, at the same time seeking the difference, and then after the implementation of the recognition of the difference between the obtained sub-areas of the image.

In the identification process focused contain edge characteristics, central feature of the linear characteristic three samples, The total of 14 fundamental characteristic of the prototype.

If the detection window size $W \times H$, With quad rectangular description:

$r=(x, y, w, h, \alpha)$, among them,$(x, y)$ is used to describe the rectangular coordinates of the vertices; $(w, h)$ is used to describe the size of a rectangle; $\alpha$ is used to describe the rotation angle of the rectangle. $\operatorname{recSum}(r)$ is used to describe graphics window and all pixels, the rectangle feature can be described as follows:

$$
\text { Feature }_{I}=\sum_{i \in I=\{1, \cdots, N\}} \omega_{i} \cdot \operatorname{recSum}\left(r_{i}\right)
$$

Where in, $\{1, \cdots, N\}$ This feature is made on behalf of two matrices; $\omega_{i}$ Continental is a graphical $i$ Weights; $\operatorname{recSum}\left(r_{i}\right)$ is all the pixels in the rectangle $i$.

Because the process of feature recognition calculation, the statistics need all the pixel values in a rectangle and makes training and testing efficiency is greatly reduced, therefore, we need to integrate special parameters to reduce property value calculating expenses, increase efficiency. Highlights include integral image angle is $0^{\circ}$ and $45^{\circ}$ the two ramps FIG. $0^{\circ}$ inclination is used below figure shows an example of integration, Transmission recSum $(r)$ evolution. For angle of $0^{\circ}$ is a characteristic square, integral image $\operatorname{SAT}(x, y)$ can be described as:

$$
\operatorname{SAT}(x, y)=\sum_{x^{\prime} \leq x, y^{\prime} \leq y} I\left(x^{\prime}, y^{\prime}\right) \text { Feature }_{1}
$$

Where in, $I\left(x^{\prime}, y^{\prime}\right)$ is used to describe the pixels value of pointer $\left(x^{\prime}, y^{\prime}\right) \cdot S A T(x, y)$ Expansion by formula (3) start, just press rows or columns to obtain a travers:

$$
\begin{aligned}
& S A T_{1}(x, y)=S A T(x, y-1)+S A T(x-1, y)+ \\
& I(x, y)-S A T(x-1, y-1)
\end{aligned}
$$

For feature matrix: $r=(x, y, w, h, 0)$, through all of the pixels of the matrix and can be obtained target identification equation:

$$
\begin{aligned}
& \operatorname{recSum}(r)=\operatorname{SAT}(x-1, y-1)+ \\
& \operatorname{SAT}(x+w-1, y+h-1)-\operatorname{SAT}(x-1, y+h-1)- \\
& \operatorname{SAT}(x+w-1, y-1)
\end{aligned}
$$

The analysis equation, matrix $r$ 's $\operatorname{recSum}(r)$. Only time will be able to obtain integral image search, computational efficiency, while high recognition rate.

\section{Identity Recognition Algorithm LBP Face Recognition Adaptive Wireless Visual Sensor Networks}

\subsection{Face Cascade Classifier}

Harr Face cascade classifier using decision tree classifier manner in each period of the implementation of the search for the face, Increase the accuracy of inspection. In each period were performed using the discrete Adaboost algorithm sample training, a set of weak and strong classifiers were selected filter. Detailed exercise procedure is as follows:

1) Consider the the number of Sample image is $N$. $\left(x_{1}, y_{1}\right),\left(x_{2}, y_{2}\right), \cdots,\left(x_{n}, y_{n}\right)$, among them, $x_{i}$ used to describe the input sample image, $y_{i}=0$ is used to describe the classical mark, in case $y_{i}=0$, Representative of the detected non-face. in case $y_{i}=1$, The representative of the detected human face samples;

2) Initialization processing by the following formula for weight:

$$
\omega_{l, i}=\left\{\begin{array}{l}
\frac{1}{2 m}, y_{i}=0 \\
\frac{1}{2 l}, y_{i}=1
\end{array}\right.
$$


Where in, $m$ and $l$ were used to describe the number of the human face of the sample, $n=m+l$;

3) By the following formula the weights are normalized:

$$
\omega_{t, i} \leftarrow \frac{\omega_{t, i}}{\sum_{j=1}^{n} \omega_{t, j}}
$$

among them, $\omega_{t}$ is used to describe the statistical distribution;

4) For any feature $j$, Train a weak classifier $h_{t}$, Calculate the error rate of $\omega_{t}$ : Equation as follows:

$$
\varepsilon_{j}=\sum_{i} \omega_{i}\left|h_{j}\left(x_{i}\right)-y_{i}\right|
$$

5) Select the minimum error rate of $\varepsilon_{t}$, classer $h_{t}$;

6) The weights are updated:

$$
\omega_{t+1, i}=\omega_{t, i} \beta_{t}^{1-e_{i}}
$$

In the formula, if $x_{i}$ Accurately classified, $e_{i}=0$, else, $e_{i}=1 ; \beta_{t}=\frac{\varepsilon_{t}}{1-\varepsilon_{t}}$.

7) The final strong classifier can be described as:

$$
h(x)=\left\{\begin{array}{l}
1, \sum_{t=1}^{T} \alpha_{t} h_{t}(x) \geq \frac{1}{2} \sum_{t=1}^{T} \log \frac{1}{\beta_{t}} \\
0, \text { else }
\end{array}\right.
$$

Harr face cascade classifier strong classifier through the implementation of the sub-window to select image input face image after image fusion under the sub-window level in continued implementation of strong classifier selection, Finally, obtained with a high accuracy rate of face sub-window image.

\subsection{Face Image Processing PCA Dimension Reduction}

Harr face cascade respectively acquires face image face range message contains a plurality of Harr-like features, you want to increase the rear face alignment efficiency shall conclude the face range characteristic vectors. PCA dimension reduction governance, to obtain a low-dimensional feature vector and maintain lower number. And then the man's face in the field after the dimensionality reduction characteristic vector is normalized control, get the person's average face. PCA dimension reduction method of face range message is as follows:

1) First, the face image of the sample by column expands into a one-dimensional vector, the calculation of their average image vector:

$$
m=\frac{1}{M} \sum_{i=1}^{M} x_{i}
$$

There in, $x_{i}$ is used to describe a face samples Vector; $M$ is used to describe the total sample; $m$ Used to describe the average vector image, in the face image of the sample it is commonly referred to as the average face.

2) Covariance matrix of training samples is calculated:

$$
C=\frac{1}{M} \sum_{i=1}^{M}\left(x_{i}-m\right)\left(x_{i}-m\right)^{T}
$$

For ease of calculation, the paper by the singular value decomposition theorems obtained eigenvalues, $\lambda_{i}(i=1,2, \cdots, n)$ used to describe, The eigen values are arranged in descending order, find the corresponding eigenvalues and orthogonal normalized eigenvectors, $\varphi_{1}, \varphi_{2}, \cdots, \varphi_{n}$ used to describe, Demand can select last $\lambda_{1} \geq \lambda_{2} \geq \cdots \geq \lambda_{n}$ Eigen face space formed, the sample can be any of a human face is projected onto the face feature space.

3) During the detection process, the input image can be projected to feature vector space face, there are:

$$
\alpha_{i}=\varphi_{i} \cdot\left(x_{i}-m\right)
$$

Where, $\alpha_{i}$ is used to describe the projection coefficients corresponding to feature vectors, which can reflect the input image feature amount of the position. Then for any image, rectangular Feature $_{1}$ reduce the dimension after dimension reduction feature can be described as:

$$
\text { Pcafeatuer }=m+\sum_{j=1}^{k} \alpha_{j} \varphi_{j}
$$

\subsection{Algorithm Implement}

LBP operator is a useful texture description operator, which uses the gray value associated with the size of the face image of each pixel with its neighbor points consisting of the binary-coded image of local texture space formed to express, not having a rotation denaturation and brightness invariant.

LBP operator can be described as follows: The name is a key point, then the size of the $3 \times 3$ window is called the gray this critical point is called the gray value of the eight-point threshold, and the associated implement contrast, if adjacent points than the center pixel value is small, the pixel position is 0 ; otherwise it is. The $3 \times 3$ binary field consisting of eight points depend on the specific rule changes for the line coding, you can get the LBP value point, this value reflects texture information in the region.

Paper completed PCA face detection and face region feature vector dimensionality reduction processing for wireless local area of the face image, which can operate LBP described as follows:

$$
\begin{aligned}
& \text { LbpFeature }\left(x_{c}, y_{c}\right)= \\
& \sum_{p=0}^{p-1} 2^{p} \cdot S\left(\text { PcaFeature }_{p}-\text { PcaFeature }_{c}\right)
\end{aligned}
$$


there in, $\left(x_{c}, y_{c}\right)$ is used to describe the facial pixel image; PcaFeature $_{c}$ is used to describe the dimensionality reduction Haar features; PcaFeature $_{p}$ used to describe the dimensionality reduction Haar feature of its neighboring points; Dimensionality reduction Harr feature is used to describe the current pixel and its neighborhood point difference formula as follows:

$$
S(x)=\left\{\begin{array}{l}
1, \text { if } \quad x \geq 0 \\
0 . \text { else }
\end{array}\right.
$$

Human face feature information $\operatorname{LbpFeature}(x, y)$ Histogram statistics, get face LBP histogram E(LbpFeature), Its visual sensor and features a wireless network of human facial feature database matching, to complete the identification of the identity. Face matching core idea is to be recognized by LBP facial features and all the characteristics of an average face library LBP features quite similar degree of variance, whether to identify suspicious face was left face characteristic library. Similarity variance by the following equation:

$$
d^{2}\left(L F_{c}, A L F_{n}\right)=\stackrel{b-1}{E}\left(L F_{c}(i), A L F_{n}(i)\right)
$$

there in, $L F_{c}$ is used to describe with face recognition information; $L F_{c}(i)$ is used to describe the face recognition LBP histogram bin characteristic features of the first block; $A L F_{n}$ and $A L F_{n}(i)$ The average face message and LBP histogram characteristics echoes; on behalf of the user to identify the person to be the face of LBP histogram bin. Similarity variance reduction, then the message with the face recognition of average face is more similar message echoes.

When the implementation of the human face LBP histogram matching, usually using a threshold to identify an empirical implementation of pairing, which is determined through variance and similarity thresholds (not the same as for practical application, by experience gains) of relations, wireless visual sensor network identity identification, as detailed below:

$$
\text { predict }=\left\{\begin{array}{l}
1, d \leq t \\
-1, d>t
\end{array}\right.
$$

LBP is based on visual characteristics of wireless sensor network identity adaptive recognition target equation can be described as:

$$
\text { faceLable }=\text { Lable }\left\{\min \left[d_{n}\left(\text { predict }_{n}=1\right)\right]\right\}
$$

Where, $\mathrm{N}$ is used to describe the number of signatures in the face of the average face; Label used to describe the average face by trained label.

\section{Simulation Analysis}

In order to verify the proposed face recognition LBP wireless visual sensor network identity effectiveness of adaptive recognition algorithm needs to implement the relevant experimental resolution. Experiment traditional gait characteristics distinguish the characteristics of identity as a comparison algorithm simulation analysis.

\subsection{Database Settings}

Here in are derived from experimental data using UATV database, experiment database 300 contains images, depicted in Figure 1 is part of a database that contains an image of the sample used in the experiment.

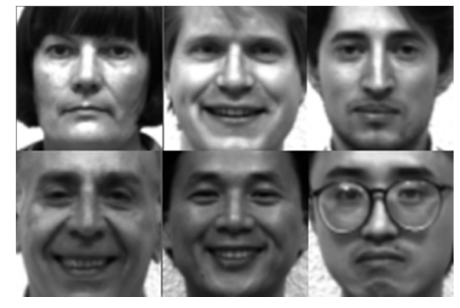

Figure 1. Experimental sample image.

\subsection{Algorithm Recognition Accuracy Analysis}

From a total match score, the average recognition rate of two aspects of the implementation of this algorithm to identify test accuracy.

Respectively, using this algorithm and the traditional algorithm for visual identity of wireless sensor networks in the implementation of adaptive identification, cumulative scores can be used to obtain matching Figure 2 will be described.

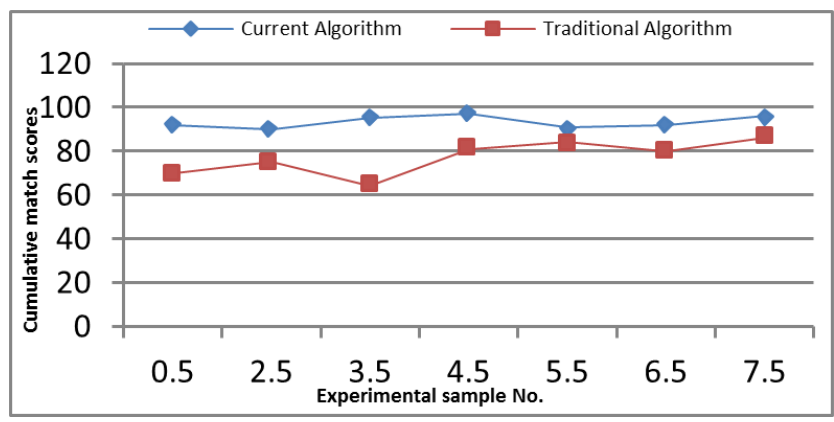

Figure 2. Cumulative match scores compare two methods results.

Analysis Figure 2 shows that the use of cumulative matching score algorithm was $94.2 \%$, the cumulative match scores traditional method is a $76.4 \%$ improvement compared to the traditional algorithm cumulative match scores improved $17.8 \%$. This is because This article describes the algorithm effective facial features, improved identification correct classification rate to verify the accuracy of the algorithm.

To further verify the accuracy of the proposed method, the average recognition accuracy of the algorithm and the 
traditional algorithm were compared and analyzed, the results shown in Figure 3.

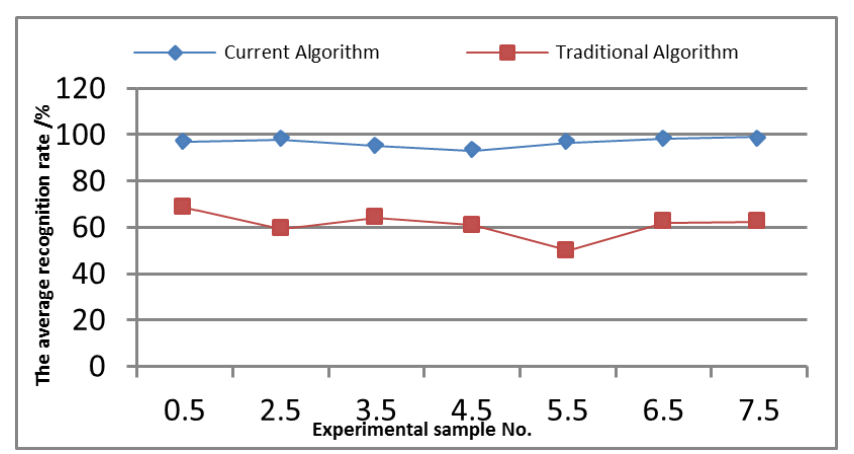

Figure 3. Two ways the average recognition accuracy of the comparison result.

Analysis can be seen in Figure 3, the algorithm recognition accuracy rate of $95.4 \%$, traditional recognition algorithm $62.7 \%$ accuracy rate. Improved algorithm and traditional algorithm, using the average recognition accuracy of this algorithm a $32.7 \%$ increase compared to the traditional method, and the average recognition accuracy of the algorithm curve is more stable, indicating that the proposed method provides high recognition accuracy, but also has very high stability.

\subsection{Algorithm Efficiency Analysis}

On different types of child face image database, respectively algorithm and traditional identity recognition algorithms, efficiency of two algorithms were compared, the results shown in:

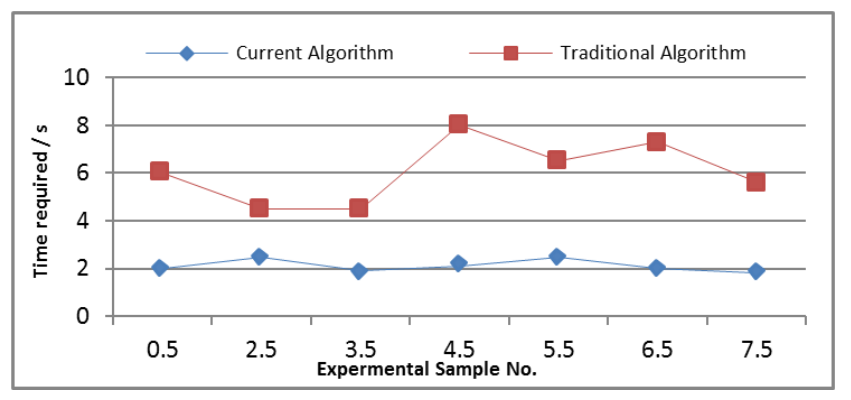

Figure 4. Comparison of the results of the two algorithms efficiency.

Analysis can be seen in Figure 4, for the same experimental sample, the algorithm time required for $2.4 \mathrm{~s}$, the time required for traditional methods of $6.3 \mathrm{~s}$. Using the time required for this algorithm compared to conventional algorithms to shorten the time required for the $3.9 \mathrm{~s}$, illustrated with traditional algorithms are compared, the higher the efficiency of the algorithm identification, is further evidence of the effectiveness of the algorithm.

\section{Conclusion}

In this paper, LBP face recognition [11] wireless visual sensor network identity adaptive recognition algorithm using
Adaboost algorithm [12] achieved strong association were cascaded devices, the use of high efficiency square integral image characteristics and the characteristics of the underlying method of extraction, after finally obtained Harr people face to face cascade classifier practice tests. Characteristics of the human face in the field of PCA dimension reduction control vector to obtain a low-dimensional features vector, and down to maintain the number of the person's average face. For wireless local area in the face image, given its operating LBP histogram statistics [13], get face LBP histogram, which feature wireless visual sensor networks and human facial feature database matching, complete identity of recognition. The simulation results show that:

1. The cumulative improvement compared with the traditional algorithm matching algorithm scores improved by $17.8 \%$;

2. The average recognition accuracy of this algorithm a $32.7 \%$ increase compared to the traditional method;

3 . The time required for the algorithm to shorten the time required for 3.9 s compared to traditional methods.

4. It can be seen that the proposed algorithm has a high accuracy and to identify efficiency.

\section{Fund Project}

2017 Zhejiang Yuexiu University of Foreign Languages Project (D2017001).

Zhejiang Province Educational Science Plan 2020 (University) Research Project, "Construction and Practice of Experimental Teaching and Management System Based on CDIO Model" (2020SCG357).

\section{References}

[1] Liu X, Li S, Kong L, et al. Feature-level Frankenstein: Eliminating Variations for Discriminative Recognition [C]. 2019 IEEE Conference on Computer Vision and Pattern Recognition (CVPR). IEEE Computer Society, 2019.

[2] Liu X, Kumar B. V. K, Yang C, et al. Dependency-aware Attention Control for Unconstrained Face Recognition with Image Sets." Proceedings of the European Conference on Computer Vision (ECCV), 2018, 548-565.

[3] Rao Y, Lu J, Zhou J. Learning Discriminative Aggregation Network for Video-Based Face Recognition and Person Re-identification [J]. International Journal of Computer Vision, 2018 (2).

[4] Hadad N, Wolf L, Shahar M. Two-Step Disentanglement for Financial Data [J]. In Proceedings of the IEEE Conference on Computer Vision and Pattern Recognition, pages 772-780, 2018.

[5] Kushwaha V, Singh M, Singh R, et al. Disguised Faces in the Wild [C]. 2018 IEEE Conference on Computer Vision and Pattern Recognition Workshops (CVPRW), 2018.

[6] Zellinger W, Grubinger T, Lughofer E, et al. Central Moment Discrepancy (CMD) for Domain-Invariant Representation Learning [C]. arXiv: 1702.08811, 2017. 
[7] Cao J, Katzir O, Jiang P, etal. DiDA: Disentangled Synthesis for Domain Adaptation [J]. arXiv preprint arXiv: 1805.08019, 2018.

[8] Li Y, Tian X, Gong M, et al. Deep Domain Generalization via Conditional Invariant Adversarial Networks [C]. European Conference on Computer Vision. 2018.

[9] Cao Q, Shen L, Xie W, et al. VGGFace2: A dataset for recognizing faces across pose and age [J]. In Automatic Face \& Gesture Recognition, 2018.

[10] Xie W, Zisserman A. Multicolumn Networks for Face Recognition [J]. ArXiv: 1807.09192, 2018.

[11] Xie W, Li S, Zisserman A. Comparator Networks [J]. In Proceedings of the European Conference on Computer Vision (ECCV), 2018.

[12] Bo Y, Chen S. A comparative study on local binary pattern (LBP) based face recognition: LBP histogram versus LBP image [J]. Neurocomputing, 2013, 120 (10): 365-379.

[13] Maze B, Adams J, Duncan JA, et al. IARPA Janus benchmark-c:
Face dataset and protocol [C]. In 2018 International Conference on Biometrics (ICB) 2018.

[14] Zeng N, Zhang H, Song B, et al. Facial expression recognition via learning deep sparse autoencoders [J]. Neurocomputing, 2017.

[15] Liu X, Vijay Kumar B. V. K, Jia P, et al. Hard negative generation for identity-disentangled facial expression recognition [J]. Pattern Recognition, 2019, 88: 1-12.

\section{Biography}

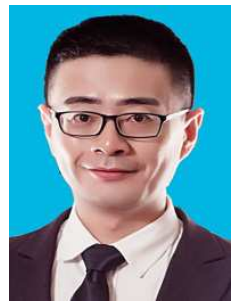

Jiangtao Wang (1980-), male, Associate Professor, Master, research direction: Graphic Image and Face Recognition 